\title{
Endothelial, inducible and neuronal nitric oxide synthase in congenital pulmonary
} lymphangiectasis

\author{
T. Hoehn*, M. William*, A.R. McPhaden`, H. Stannigel*, \\ E. Mayatepek* and R.M. Wadsworth ${ }^{+}$
}

ABSTRACT: Abnormal growth and development of lymphatic pulmonary structures leads to severe hypoxia in congenital pulmonary lymphangiectasis (CPL).

This case study aims to determine the cellular source and topographical distribution of the nitric oxide synthases in CPL. It studies the post mortem tissue of a term newborn with the clinical course and histological findings of CPL and three controls without pulmonary pathology.

It was found that endothelial cells of pulmonary arteries and lymphatic structures stained significantly more for endothelial nitric oxide synthase protein in the CPL patient compared to the controls.

The authors conclude that synthesis of endothelial nitric oxide synthase is upregulated in vascular and lymphatic endothelial cells in congenital pulmonary lymphangiectasis.

KEYWORDS: Endothelial cells, endothelial nitric oxide synthase, inducible nitric oxide synthase, inhaled nitric oxide, newborn, nitrotyrosine

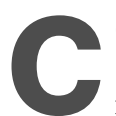

ongenital pulmonary lymphangiectasis (CPL) is a rare disease of the term newborn, which was originally described by R. Virchow almost 150 yrs ago [1, 2]. Congenital dilatation of the pulmonary lymphatic vessels is the key feature of this disease and has been classified according to its presence in other organs and the existence of associated malformations [3]. In the majority of cases, cyanosis is present shortly after birth, though diagnosis can be delayed for several weeks in cases of unilobar presentation [4]. Mortality is higher if both lungs are affected [5], and early foetal signs can be those of non-immune hydrops foetalis [6]. Congenital anomalies (cardiac and renal) have been reported in $>50 \%$ of cases of CPL [5], and in one case, CPL was caused by thoracic duct agenesis [7]. Although primarily a disease of the term newborn, CPL can present in the pre-term infant and spontaneous resolution of the condition can occur [8].

This study describes the findings of immunohistochemical staining for the nitric oxide synthases (NOS), endothelial NOS (eNOS), inducible NOS (iNOS) and neuronal NOS (nNOS) in a term newborn with CPL.

\section{MATERIAL AND METHODS}

\section{Case report}

The pregnancy of a 37-yr-old primigravida was induced by intracytoplasmic sperm injection; the course of the pregnancy was entirely uneventful. Spontaneous vaginal delivery took place after 41 weeks and specific risk factors for either maternal or neonatal infection were absent. The amount of amniotic fluid and the macroscopic appearance of the placenta were normal. Arterial cord blood $\mathrm{pH}$ was 7.27 , birth weight was $3,400 \mathrm{~g}$, APGAR was 8/8/8 at 1, 5 and $10 \mathrm{~min}$, and there were no obvious malformations or dysmorphic signs. Initial cyanosis and bradycardia persisted post-natally; neither were substantially improved by bag and mask ventilation with $100 \%$ oxygen. On arrival of the neonatal transport team the infant was on nasopharyngeal continuous positive airway pressure with $100 \%$ oxygen; maximum transcutaneous oxygen saturation by pulse oxymetry was $50 \%$. Following endotracheal intubation the heart rate increased to normal levels and oxygen saturation did not improve to $>60 \%$. The latter stayed at this level during transport to the neonatal intensive care unit (Dept of General Paediatrics, Heinrich-Heine-University,
AFFILIATIONS

*Neonatology and Paediatric Intensive Care Medicine, Dept of General Paediatrics, and

${ }^{\#}$ Dept of Pathology, Heinrich-HeineUniversity, Düsseldorf, Germany.

"Dept of Pathology, Glasgow Royal Infirmary, and

${ }^{+}$Dept of Physiology and Pharmacology, University of Strathclyde, Glasgow, UK.

CORRESPONDENCE

T. Hoehn

Neonatology and Paediatric Intensive Care Medicine

Dept of General Paediatrics

Heinrich-Heine-University

Moorenstr. 5

D-40225 Düsseldorf Germany

Fax: 492118119786

E-mail: thomas.hoehn@uniduesseldorf.de

Received:

July 152005

Accepted after revision:

November 052005

European Respiratory Journal Print ISSN 0903-1936 Online ISSN 1399-3003 
Düsseldorf, Germany) and respiratory parameters were as follows: peak inspiratory pressure $2.5 \mathrm{kPa}$, positive endexpiratory pressure $0.5 \mathrm{kPa}$, rate $63 \cdot \mathrm{min}^{-1}$ and inspiratory oxygen fraction 1.0. At this stage the chest radiograph showed inhomogenous, streaky infiltrates bilaterally and a small leftsided pneumothorax. Echocardiography revealed normal cardiac anatomy and showed pronounced pulmonary hypertension as quantified by severe tricuspid incompetence. Therapy consisted of antibiotic treatment, ventilation, inhaled nitric oxide (NO; up to $35 \mathrm{ppm}$ ), volume expansion, sodium bicarbonate and catecholamines. Despite high doses of catecholamines resulting in adequate blood pressure and continuous infusion of sodium bicarbonate, respiratory as well as metabolic acidosis increased. Left-sided tension pneumothorax was diagnosed and relieved by a chest tube. Terminal bradycardia non-responsive to catecholamine therapy occurred after $9 \mathrm{~h}$ of life and the infant died. Autopsy reported cystic CPL confined to the lungs.

\section{Controls}

Control subjects were chosen from infants who had died from sudden infant death syndrome and had an autopsy performed at the Dept of Paidopathology and Placentology of Humboldt Univesity (Charité Virchow Hospital, Berlin, Germany). None of the infants was resuscitated by medical personnel, there were no therapeutic interventions (e.g. mechanical ventilation) nor was there evidence of pulmonary infection in any of the infants.

\section{Control 1}

Infant of a 15-yr-old primigravida, with maternal cigarette smoking. The term infant had a birth weight of $3,000 \mathrm{~g}$, an APGAR of $9 / 10$ and an arterial cord $\mathrm{pH}$ of 7.29. Diarrhoea was noted 10 days prior to death. The infant died on day 73 of life.

\section{Control 2}

Infant of a 22-yr-old primigravida, with a history of smoking. The term infant had a birth weight of 2,740 g, an APGAR of 8/ 10 and an arterial cord $\mathrm{pH}$ of 7.24. The infant died on day 86 of life.

\section{Control 3}

Infant from the second pregnancy of a 24-yr-old mother, with a history of smoking. Delivery was at 39 weeks of gestation and the infant had a birth weight of 2,610 g, meconium-stained amniotic fluids, an APGAR of $9 / 9$ and an arterial cord $\mathrm{pH}$ of 7.29. Local fungal infection of mouth and skin occurred at 8 weeks of age. The infant died on day 137 of life.

\section{Methodology for immunocytochemistry}

Lung tissue was supplied as paraffin-embedded tissue blocks. Sections $(4 \mu \mathrm{m})$ were cut from the blocks, rehydrated and then antigen was retrieved by microwave pressure cooking or trypsin incubation. The sections were treated to block nonspecific binding of primary and secondary antibodies and nonspecific reaction with chromogens as described previously [9]. The sections were then incubated with the specific antibody for $60 \mathrm{~min}$ at room temperature (eNOS: catalogue No. N30020, BD Transduction, Lexington, KY, USA; 1:200; pretreatment was pressure cooking. iNOS: Cat. No. N32020, BD Transduction; 1:500; pre-treatment was pressure cooking.
nNOS: Cat. No. 610308, BD Biosciences, Oxford, UK; 1:400; pre-treatment was pressure cooking. Nitrotyrosine: Cat. No. 06-284, Upstate Biotechnology, Lake Placid, NY, USA; 1:200; pre-treatment was trypsin incubation). Nitrotyrosine staining was included in order to verify whether or not sufficient NO was formed to induce the formation of peroxynitrite, as indicated by tyrosine nitration. Bound antibody was detected using goat anti-mouse immunoglobulin $G$ conjugated with horseradish peroxidase using a streptavidinbiotin link and visualised with diaminobenzidine. In negative controls, the primary antibody was replaced with pre-immune serum. Sections were counterstained using haematoxylin and viewed by light microscopy.

Vessels with an internal diameter $<250 \mu \mathrm{m}$ were regarded as small pulmonary arteries, whereas those $>250 \mu \mathrm{m}$ were regarded as large pulmonary arteries.

\section{RESULTS \\ Endothelial nitric oxide synthase}

Moderate endothelial positivity for eNOS was found in medium-sized pulmonary arteries and in small pulmonary artery branches (fig. 1a), the latter usually being negative for eNOS. There was also mild positive staining of the endothelial lining of some of the ectatic lymphatic channels.

In the controls there was slight positivity in the endothelium of the large arteries, but none was visible in the small arteries (fig. 1b).

\section{Inducible nitric oxide synthase}

Mild-to-moderate endothelial positivity was present in medium and small pulmonary artery branches, which also showed some smooth muscle staining (fig. 2a). Some of the ectatic lymphatic channels showed mild endothelial positivity, although in some areas the endothelial lining had been lost. Moderate positivity was also present in bronchial epithelial cells and mild positivity was seen in alveolar lining cells.

The controls had a similar staining pattern, and thus there was no overall upregulation of iNOS in CPL compared to controls (fig. 2b).

\section{Neuronal nitric oxide synthase}

Lymphatic and pulmonary artery structures had no convincing positive staining in either the patient or the controls (fig. 3a and $b$ ). nNOS was detected in the airway epithelium in both groups.

\section{Nitrotyrosine}

High background made interpretation difficult but there appeared to be bronchial epithelial cell and interstitial positivity. The endothelium of the ectatic lymphatic channels was more convincingly positive than the blood vessel endothelium.

In controls, the media of pulmonary arteries stained lightly for nitrotyrosine.

Staining details have been summarised in table 1 .

\section{DISCUSSION}

Induction of eNOS synthesis in endothelial cells of small- and medium-sized pulmonary arteries has been shown in a term 

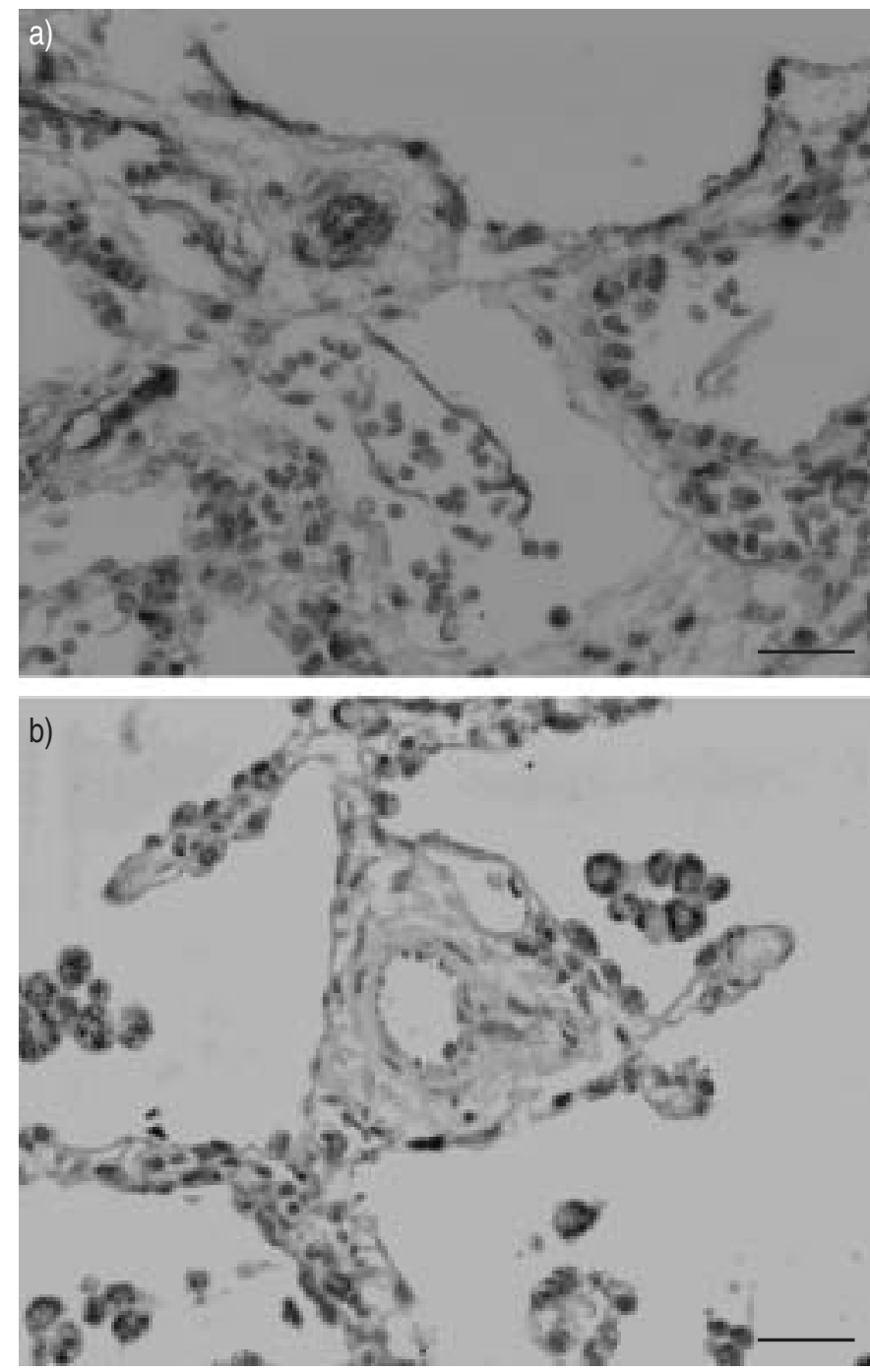

FIGURE 1. a) Haematoxylin and eosin-stained immunohistochemistry of paraffin-embedded lung tissue sample for endothelial nitric oxide synthase (eNOS) in the congenital pulmonary lymphangiectasis patient showing positive staining of the endothelial lining of the ectatic lymphatic channel at the top of the photomicrograph. There is also strong endothelial positivity of the prominent small pulmonary artery branch seen immediately below this lymphatic channel. Endothelial positivity of thinner walled vascular channels is also present elsewhere along with some nonspecific erythrocyte positivity. b) Haematoxylin and eosin-stained immunohistochemistry for eNOS in a control infant showing very mild positive staining, only of the endothelial lining of the small pulmonary arteries. Scale bars $=100 \mu \mathrm{m}$.

newborn with CPL. eNOS synthesis was also present in the endothelial lining of the ectatic lymphatic structures in this patient. To the present authors' knowledge this is the first report of immunohistochemistry findings of the NOS enzyme family in neonatal CPL.

Although not uniformly a fatal disease [10-12], neonatal presentation of bilateral CPL is associated with severe compromise of pulmonary gas exchange as well as high mortality. Neither clinical presentation nor macroscopic aspect during surgery allow definite differentiation between CPL and congenital lobar emphysema [13] or between CPL and pulmonary lymphangioma [14]. Extrapulmonary manifestation of
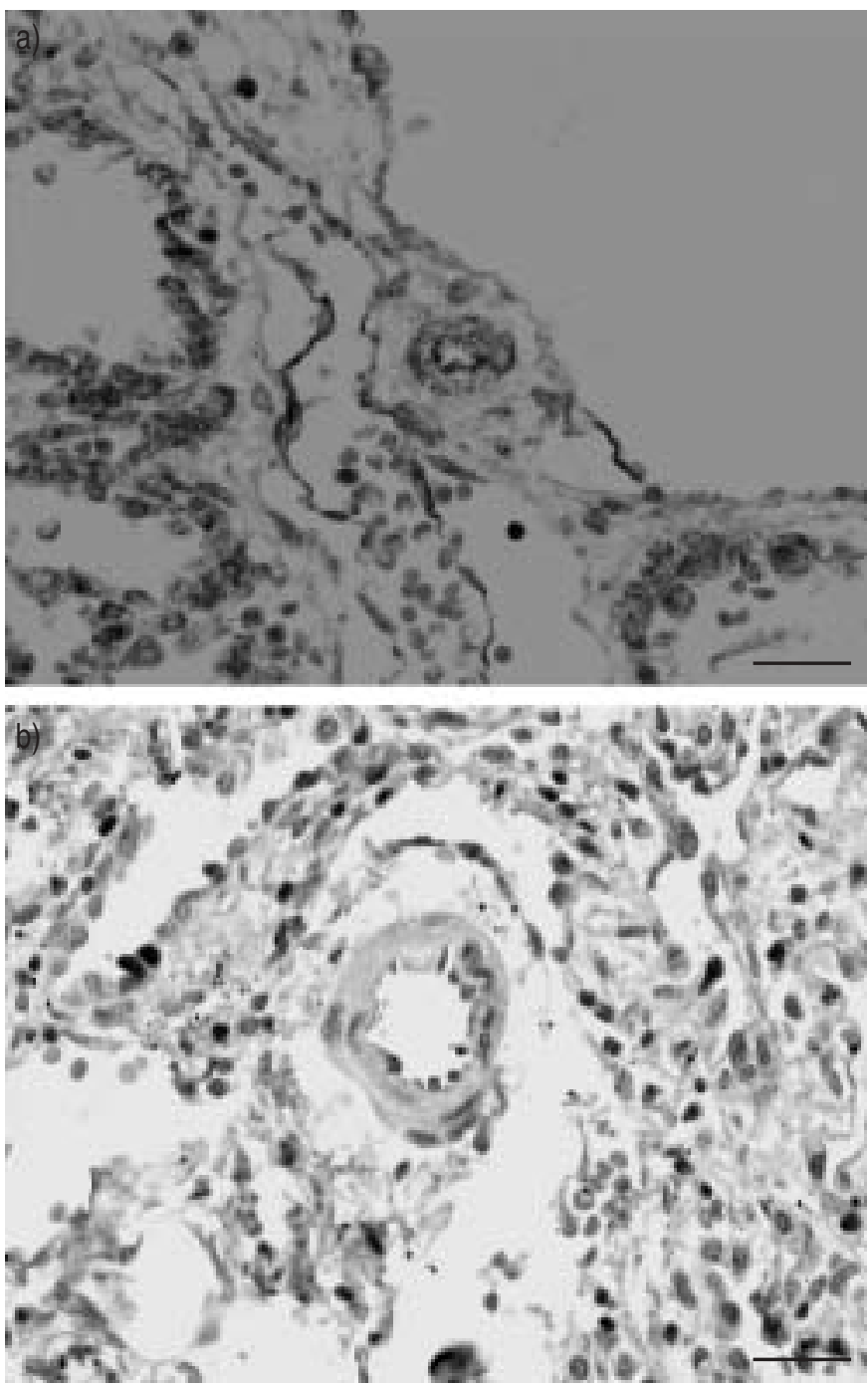

FIGURE 2. a) Haematoxylin and eosin-stained immunohistochemistry of paraffin-embedded lung tissue samples for inducible nitric oxide synthase (iNOS) in the congenital pulmonary lymphangiectasis patient showing positive staining of the endothelial lining of ectatic lymphatic channels occupying the top right of the image and snaking through the centre of the image orientated vertically. Sandwiched between them is a prominent small pulmonary artery branch demonstrating endothelial positivity. Positive alveolar lining cells are present down the left-hand side and in the bottom right. b) Haematoxylin and eosin stain of the control's small pulmonary artery showing no significant iNOS positivity. Intraalveolar macrophages positive. Scale bars $=100 \mu \mathrm{m}$.

lymphangiectasis is not necessarily associated with better pulmonary gas exchange [15]. Recently, the present authors have shown upregulation of eNOS in patients with rapid progressive pulmonary hypertension of the newborn (RPPHN) [9]. Hypoxia could be expected to act as a trigger for eNOS upregulation both in RPPHN as well as in CPL. The main difference between RPPHN and CPL regarding eNOS upregulation is the time axis: whereas infants with RPPHN were several weeks of age at the time of biopsy or autopsy, the infant with CPL was only $9 \mathrm{~h}$ of age. One possible explanation for the early upregulation of eNOS in the present case could be the degree of hypoxia. Arterial partial pressures of oxygen in the present patient were 

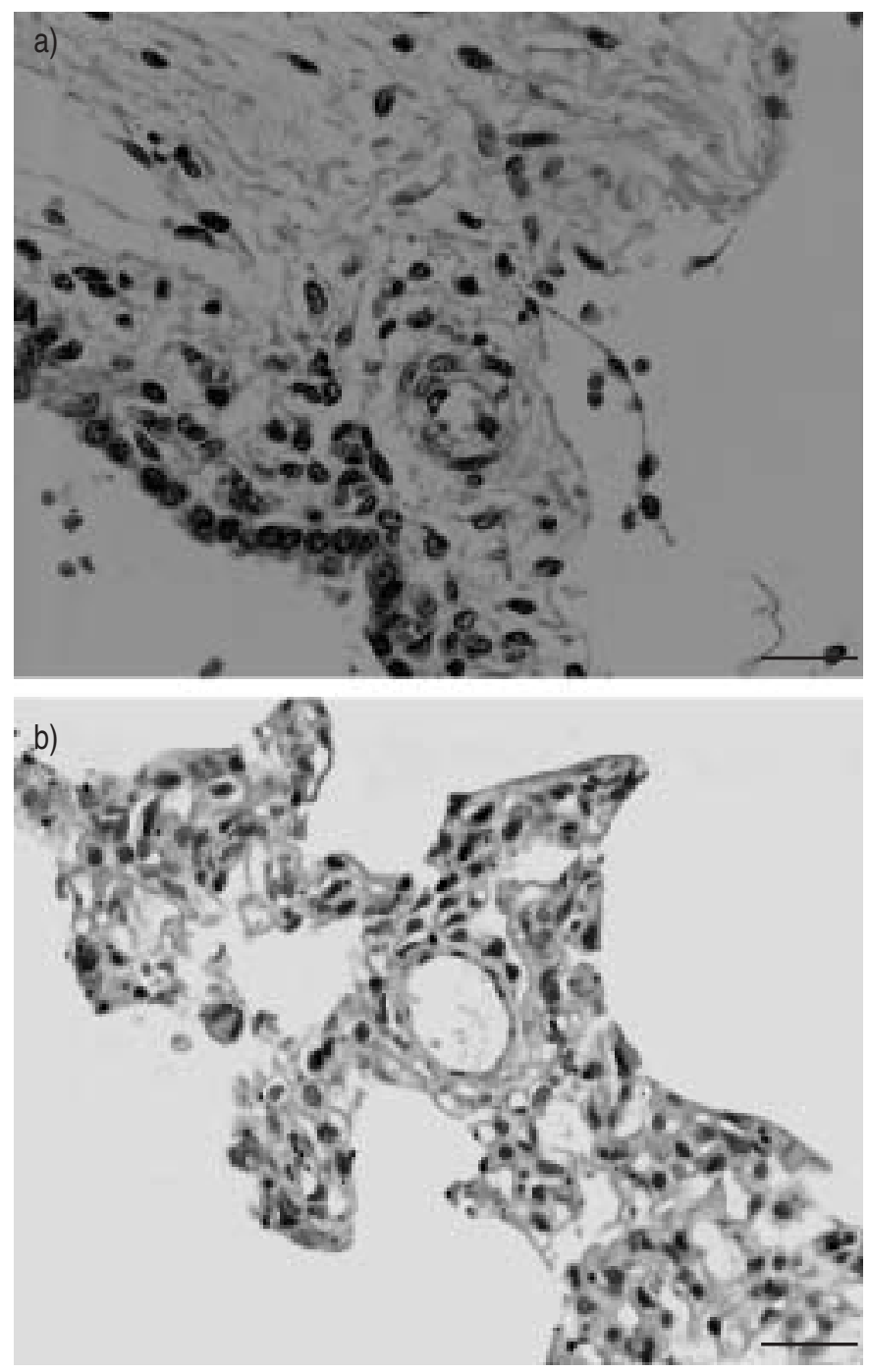

FIGURE 3. a) Haematoxylin and eosin-stained immunohistochemistry of paraffin-embedded lung tissue samples for neuronal nitric oxide synthase (nNOS) in the congenital pulmonary lymphangiectasis patient showing mild positivity in respiratory epithelial cells only. The small pulmonary artery branch shown is negative. b) Haematoxylin and eosin stain of the control's small pulmonary artery showing no positivity by immunocytochemistry for nNOS. Scale bars $=100 \mu \mathrm{m}$.
1.2-3.2 $\mathrm{kPa}$ and therefore lower than normal foetal intrauterine oxygen levels (which range 3.6-4.7 $\mathrm{kPa}$ ) [16].

The second interesting finding consisted of eNOS expression in lymphatic endothelial cells, which are so prominent and pathogenic in CPL. Earlier in vitro investigations have established a model for the study of microlymphatics [17] and have provided evidence of NO production by lymphatic endothelial cells [18]. Depending on the type of stimulus (Ca ionophore or lipopolysaccharide), NO production was mediated by eNOS or by iNOS upregulation [19]. In vivo studies in C57BL/6 mice have shown that eNOS affects lymphatic fluid flow without affecting the diameter of the initial lymphatics [20]. Lymphatic flow appears to be regulated by the collecting lymphatics and the degree of outflow resistance, which was regulated by eNOS.

Upregulation of iNOS does not appear to be a feature of CPL. In a rat model of pulmonary hypertension, initial upregulation of iNOS subsided after several days, leaving upregulation of eNOS the predominant finding [21].

Although there is reason to believe that treatment with $\mathrm{NO}$ does not influence eNOS expression, this possibility cannot be excluded. At least in a rat model of pulmonary hypertension, the addition of $\mathrm{NO}$ to the study gas did not modify pulmonary eNOS expression [22].

At this stage it can only be speculated what effect endothelial nitric oxide synthase upregulation in vascular endothelial cells and in lymphatic endothelial cells has on the resulting degree of hypoxia. Nitric oxide formation would result in vasodilation with reduced vascular resistance, increased pulmonary blood flow and improved oxygenation in the case of vascular endothelial cells. Vasodilation in pulmonary lymphatics could mean less outflow resistance, improved pulmonary clearance of lymphatic fluids and, again, improved oxygenation. In the present case at least, not even the addition of exogenous nitric oxide by means of inhalation was sufficient to reverse profound hypoxia nor to prevent severe acidosis and ultimately, death.

\section{ACKNOWLEDGEMENTS}

The authors are grateful for the support they received from $\mathrm{H}$. Müntefering in establishing the histological diagnosis of

\begin{tabular}{|c|c|c|c|}
\hline eNOS & $\begin{array}{l}\text { CPL. Medium-sized pulmonary arteries, small pulmonary } \\
\text { artery branches }\end{array}$ & $\begin{array}{l}\text { Controls. Endothelium of large pulmonary } \\
\text { arteries }\end{array}$ & Controls. Small pulmonary arteries \\
\hline \multirow[t]{2}{*}{ iNOS } & $\begin{array}{l}\text { CPL. Small and medium pulmonary arteries, } \\
\text { smooth muscle, bronchial epithelial cells }\end{array}$ & CPL. Alveolar lining cells & \\
\hline & $\begin{array}{l}\text { Controls. Small and medium pulmonary arteries, } \\
\text { smooth muscle, bronchial epithelial cells }\end{array}$ & Controls. Alveolar lining cells & \\
\hline nNOS & & CPL. Airway epithelium & $\begin{array}{l}\mathrm{CPL} \text { and controls. Lymphatic and } \\
\text { pulmonary artery structures }\end{array}$ \\
\hline
\end{tabular}


congenital pulmonary lymphangiectasis. They would also like to thank A.A. Preston for preparing and staining the specimens immunhistochemically.

\section{REFERENCES}

1 Virchow R. Gesammelte Abhandlungen zur wissenschaftlichen Medicin. [Collected Essays on Scientific Medicine.] Frankfurt am Main, Meidinger Sohn \& Co., 1856; p. 982.

2 Laurence KM. Congenital pulmonary cystic lymphangiectasis. J Pathol Bacteriol 1955; 70: 325-333.

3 Noonan JA, Walters LR, Reeves JT. Congenital pulmonary lymphangiectasis. Am J Dis Child 1970; 120: 314-319.

4 Rettwitz-Volk W, Schlosser R, Ahrens P, Horlin A. Congenital unilobar pulmonary lymphangiectasis. Pediatr Pulmonol 1999; 27: 290-292.

5 Fronstin MH, Hooper GS, Besse BE, Ferreri S. Congenital pulmonary cystic lymphangiectasis. Case report and a review of 32 cases. Am J Dis Child 1967; 114: 330-335.

6 Njolstad PR, Reigstad H, Westby J, Espeland A. Familial non-immune hydrops fetalis and congenital pulmonary lymphangiectasia. Eur J Pediatr 1998; 157: 498-501.

7 Antonetti M, Manuck TA, Schramm C, Hight D. Congenital pulmonary lymphangiectasia: a case report of thoracic duct agenesis. Pediatr Pulmonol 2001; 32: 184-186.

8 Scott C, Wallis C, Dinwiddie R, Owens C, Coren M. Primary pulmonary lymphangiectasis in a premature infant: resolution following intensive care. Pediatr Pulmonol 2003; 35: 405-406.

9 Hoehn T, Preston AA, McPhaden AR, et al. Endothelial nitric oxide synthase (NOS) is upregulated in rapid progressive pulmonary hypertension of the newborn. Intensive Care Med 2003; 29: 1757-1762.

10 Hagmann C, Berger TM. Images in clinical medicine. Congenital pulmonary lymphangiectasia. $N$ Engl J Med 2003; 349: e21.

11 Finder J, Steinfeld J. Congenital pulmonary lymphangiectasia. N Engl J Med 2004; 350: 948.
12 Bouchard S, Di Lorenzo M, Youssef S, Simard P, Lapierre JG. Pulmonary lymphangiectasia revisited. J Pediatr Surg 2000; 35: 796-800.

13 Chapdelaine J, Beaunoyer M, St-Vil D, et al. Unilobar congenital pulmonary lymphangiectasis mimicking congenital lobar emphysema: an underestimated presentation? J Pediatr Surg 2004; 39: 677-680.

14 Brown M, Pysher T, Coffin CM. Lymphangioma and congenital pulmonary lymphangiectasis: a histologic, immunohistochemical, and clinicopathologic comparison. Mod Pathol 1999; 12: 569-575.

15 Hirano H, Nishigami T, Okimura A, Nakasho K, Uematsu K. Autopsy case of congenital pulmonary lymphangiectasis. Pathol Int 2004; 54: 532-536.

16 Mildenberger E, Siegel G, Versmold HT. Oxygen-dependent regulation of membrane potential and vascular tone of human umbilical vein. Am J Obstet Gynecol 1999; 181: 696-700.

17 Hayes H, Kossmann E, Wilson E, Meininger C, Zawieja D. Development and characterization of endothelial cells from rat microlymphatics. Lymphat Res Biol 2003; 1: 101-119.

18 Robertson DA, Hughes GA, Lyles GA. Expression of inducible nitric oxide synthase in cultured smooth muscle cells from rat mesenteric lymphatic vessels. Microcirculation 2004; 11: 503-515.

19 Leak LV, Cadet JL, Griffin CP, Richardson K. Nitric oxide production by lymphatic endothelial cells in vitro. Biochem Biophys Res Commun 1995; 217: 96-105.

20 Hagendoorn J, Padera TP, Kashiwagi S, et al. Endothelial nitric oxide synthase regulates microlymphatic flow via collecting lymphatics. Circ Res 2004; 95: 204-209.

21 Hampl V, Bibova J, Banasova A, et al. Pulmonary vascular iNOS induction participates in the onset of chronic hypoxic pulmonary hypertension. Am J Physiol Lung Cell Mol Physiol 2006; 290: L11-L20.

22 Lin YJ, Markham NE, Balasubramaniam V, et al. Inhaled nitric oxide enhances distal lung growth after exposure to hyperoxia in neonatal rats. Pediatr Res 2005; 58: 22-29. 